Research Article; Received: March 4, 2021; Accepted: July 9, 2021

\title{
AN INVESTIGATION ON THE TRIPLE IDEAL CONVERGENT SEQUENCES IN FUZZY METRIC SPACES
}

\author{
Mehmet GÜRDAL ${ }^{1}$ and Ekrem SAVAŞ ${ }^{2}$ \\ ${ }^{1}$ Suleyman Demirel University, Department of Mathematics, 32260 Isparta, TURKEY \\ ${ }^{2}$ Uşak University, 64100 Uşak, TURKEY
}

\begin{abstract}
The notion of ideal convergence is a process of generalizing of statistical convergence which is dependent on the idea of the ideal $\mathcal{I}$ of subsets of the set positive integer numbers. In this study we also present the concept of ideal convergence for triple sequences in fuzzy metric spaces (FMS) in the manner of George and Veeramani and the terms of ideal Cauchy sequence and $\mathcal{I}^{*}$-Cauchy sequence in FMS and examine their some properties.
\end{abstract}

\section{Introduction and Literature Review}

Statistical convergence for real sequence was rst introduced by Fast [4] in 1951 . Since then statistical convergence was investigated by more and more researchers. The concept of $\mathcal{I}$-convergence, and interesting generalization of statistical convergence [4], was first presented by Kostyrko et al. 20] with use of the ideal $\mathcal{I}$ of subsets of the set of natural numbers $\mathbb{N}$ and further studies done in 27 . The study of ideal convergence in triple sequence has been initiated by Şahiner and Tripathy [31. More analysis in this field and more implications of these statistical convergence and ideal convergence can be seen in $1,11,13,15,22,24,26,28,32,36,39,40]$.

After Zadeh's leading work in 1965, fuzzy set theory has been widely applied into practical problems. Fuzzy set theory is a very effective set for modelling uncertainty and vagueness in various problems that arise in some fields. Many authors have defined several concepts of FMS in different ways [3, 5, 16, 18, 21, 23. In [5, 6, George and Veeramani first investigated and presented the notion of fuzzy metric space with the use of continuous t-norms. Lately, several convergences in fuzzy metric spaces were studied by Gregori et al. $7-10$.

2020 Mathematics Subject Classification. Primary 40A05; Secondary 54A40, 54D35, 54E50.

Keywords. Ideal Cauchy sequence, Fuzzy metric, triple sequence, ideal convergence.

घurdalmehmet@sdu.edu.tr-Corresponding author; ekremsavas@yahoo.com

(D) 0000-0003-0866-1869; 0000-0003-2135-3094. 
Generally, statistically convergent sequences fulfills most of the features of ordinary convergent sequences in metric spaces. For example, a statistically convergent sequence is statistically Cauchy ( 29]) in an arbitrary metric space. Concordantly, we introduce studying $\mathcal{I}$-Cauchy and $\mathcal{I}$-convergence concepts of triple sequences on FMS.

Here, as it can be recalled the following basic concepts from 2, 5, 18, 38, needed in the course of the paper.

Definition 1. The 3 -tuple $(X, \mathcal{M}, *)$ is said to be a FMS if $X$ is a nonempty set, * is a continuous $t$-norm and $\mathcal{M}$ is a fuzzy set on $X^{2} \times(0, \infty)$ satisfying the following cases for all $x, y, z \in X$ and $s, t>0$ :

Case 1. $\mathcal{M}(x, y, t)>0$

Case 2. $\mathcal{M}(x, y, t)=1$ iff $x=y$;

Case 3. $\mathcal{M}(x, y, t)=\mathcal{M}(y, x, t)$;

Case 4. $\mathcal{M}(x, y, t) * \mathcal{M}(y, z, s) \leq \mathcal{M}(x, z, t+s)$

Case 5. $\mathcal{M}(x, y,):.(0, \infty) \rightarrow[0,1]$ is continuous.

Definition 2. Let $(X, \mathcal{M}, *)$ be a FMS. We define open ball $B_{\mathcal{M}}(x, r, t)$ with centre $x \in X$ and radius $r, 0<r<1, t>0$ as

$$
B_{\mathcal{M}}(x, r, t)=\{y \in X: \mathcal{M}(x, y, t)>1-r\} .
$$

Let $(X, \mathcal{M}, *)$ be a FMS. We have

$\tau_{\mathcal{M}}=\left\{A \subset X: x \in A\right.$ iff there exists $t>0, r \in(0,1)$ such that $\left.B_{\mathcal{M}}(x, r, t) \subset A\right\}$.

Hence $\tau_{\mathcal{M}}$ is a topology on $X$. George and Veeramani 5 proved that $\left\{B_{\mathcal{M}}(x, r, t)\right.$ : $x \in X, t>0, r \in(0,1)\}$ forms a base of a topology $\tau_{\mathcal{M}}$ in $X$.

Definition 3. Let $(X, \mathcal{M}, *)$ be a FMS. If for every $r \in(0,1)$ and $t>0$, there exists $n_{0} \in \mathbb{N}$ such that $\mathcal{M}\left(x_{n}, x_{0}, t\right)>1-r$ for all $n>n_{0}$, then a sequence $\left\{x_{n}\right\}$ converges to $x_{0}$.

Definition 4. A sequence $\left\{x_{n}\right\}$ in a FMS $(X, \mathcal{M}, *)$ is called to be a Cauchy sequence if for all $\varepsilon, 0<\varepsilon<1$ and $t>0$, there exists $n_{0} \in \mathbb{N}$ such that $\mathcal{M}\left(x_{n}, x_{m}, t\right)>1-\varepsilon$ for every $n, m \geq n_{0}$.

Definition 5. When every Cauchy sequence is convergent, a FMS is called to be complete.

Definition 6. ([4]) Let $A \subset \mathbb{N}$, put $A_{n}=\{k \in A: k \leq n\}, \forall n \in \mathbb{N}$. Then

$$
\bar{\delta}(A):=\limsup _{n \rightarrow \infty} \frac{\left|A_{n}\right|}{n} \text { and } \underline{\delta}(A):=\liminf _{n \rightarrow \infty} \frac{\left|A_{n}\right|}{n}
$$

are called upper and lower asymptotic density of the set $A$, respectively. When $\bar{\delta}(A)=\underline{\delta}(A)$,

$$
\delta(A):=\lim _{n \rightarrow \infty} \frac{\left|A_{n}\right|}{n}
$$


is called to be an asymptotic density of $A$. All the three densities, if they exist, are in $[0,1]$.

Utilizing above information, we recall that a sequence $\left(x_{k}\right)_{k \in \mathbb{N}}$ is statistical convergent to $x$, if for all $\varepsilon>0$,

$$
\delta\left(\left\{k \in \mathbb{N}:\left|x_{k}-x\right| \geq \varepsilon\right\}\right)=0 .
$$

If $\left(x_{k}\right)_{k \in \mathbb{N}}$ is statistically convergent to $x$, we show $s t-\lim x_{k}=x$.

The terms of statistical convergence and statistical Cauchy for sequences in FMS have been investigated by $\mathrm{Li}$ et al. [19].

Definition 7. Let $(X, \mathcal{M}, *)$ be a FMS. if for all $r \in(0,1)$ and $t>0$

$$
\delta\left(\left\{n \in \mathbb{N}: \mathcal{M}\left(x_{n}, x_{0}, t\right)>1-r\right\}\right)=1,
$$

then a sequence $\left\{x_{n}\right\}$ in $X$ is called statistically convergent to $x_{0} \in X$

Definition 8. Let $(X, \mathcal{M}, *)$ be a FMS. If for every $r \in(0,1)$ and $t>0$, there exists $N_{0} \in \mathbb{N}$ such that

$$
\delta\left(\left\{k \in \mathbb{N}: \mathcal{M}\left(x_{k}, x_{N_{0}}, t\right)>1-r\right\}\right)=1 .
$$

A sequence $\left\{x_{n}\right\}$ in $X$ is called a statistically Cauchy sequence.

Also, Sahiner et al. 30 investigated the statistical convergence for triple sequence. A function $x: \mathbb{N}^{3}=\mathbb{N} \times \mathbb{N} \times \mathbb{N} \rightarrow \mathbb{R}$ is said to be a real triple sequence. A triple sequence $\left(x_{n k l}\right)$ in $\mathbb{R}$ is called to be converge if there exists a point $\ell$ such that for all $\varepsilon>0$, there exists a positive integer $n_{0}$ such that $\left|x_{n k l}-\ell\right|<\varepsilon$ for all $n, k, l \geq n_{0}$.

Definition 9. If

$$
\delta_{3}(A)=\lim _{n, k, l \rightarrow \infty} \frac{\left|A_{n k l}\right|}{n k l}
$$

exists, then a subset $A$ of $\mathbb{N}^{3}$ is called to have natural density $\delta_{3}(A)$. From here, if for every $\varepsilon>0$

$$
\delta_{3}\left(\left\{(n, k, l) \in \mathbb{N}^{3}:\left|x_{n k l}-\ell\right| \geq \varepsilon\right\}\right)=0,
$$

then a real triple sequence $x=\left(x_{n k l}\right)$ is called to be statistically convergent to $\ell$

Then, we give the terms of lacunary statistical convergence and lacunary statistical Cauchy for triple sequences in FMS as follows.

Definition 10. Let $(X, \mathcal{M}, *)$ be a FMS and $\theta_{3}=\theta_{r, s, t}$ be a lacunary triple sequence. A triple sequence $\left\{x_{j k l}\right\}$ is called to be lacunary statistically convergent to $\ell \in X$, written as $\operatorname{sts}_{\theta_{3}}-\lim x_{j k l}=\ell$, if, for all $r \in(0,1)$ and $t>0$,

$$
\lim _{r, s, t} \frac{1}{h_{r, s, t}}\left|\left\{(j, k, l) \in I_{r, s, t}: \mathcal{M}\left(x_{j k l}, \ell, t\right)>1-r\right\}\right|=1 .
$$


Definition 11. Let $(X, \mathcal{M}, *)$ be a FMS and $\theta_{3}=\theta_{r, s, t}$ be a lacunary triple sequence. A triple sequence $\left\{x_{j k l}\right\}$ in $X$ is said to be lacunary statistically Cauchy sequence, if, for all $\alpha \in(0,1)$ and $t>0$, there exists $\mathcal{M}, \mathcal{M}^{\prime}, \mathcal{M}^{\prime \prime} \in \mathbb{N}$ such that for all $j, p \geq \mathcal{M}^{\prime \prime}, k, q \geq \mathcal{M}^{\prime}, l, r \geq \mathcal{M}$,

$$
\delta_{\theta_{3}}\left(\left\{(j, k, l) \in \mathbb{N}^{3}: \mathcal{M}\left(x_{j k l}, x_{p q r}, t\right)>1-\alpha\right\}\right)=1 .
$$

We recall the following some notations used in 20,27.

Definition 12. A class $\mathcal{I} \subset 2^{R}$ of subsets of a nonempty set $R$ is called to be an ideal in $R$ if (i) $\emptyset \in \mathcal{I}$; (ii) $M, N \in \mathcal{I}$ imply $M \cup N \in \mathcal{I}$; (iii) $M \in \mathcal{I}, N \subset M$ imply $N \in \mathcal{I}$. A non-trivial ideal $\mathcal{I}$ in $R$ is called an admissible ideal if it is different from $P(\mathbb{N})$ and it contains all singletons, that is, $\{x\} \in I$ for each $x \in R$.

Lemma 1. Let $\mathcal{I}$ be a proper ideal in $R$, so $R \notin \mathcal{I}, R \neq \emptyset$. Then the class of sets

$$
\mathcal{F}(\mathcal{I})=\{A \subset R: \exists M \in \mathcal{I}: A=R \backslash M\}
$$

is a filter in $R$. It is said to be the filter associated with the ideal $\mathcal{I}$.

Definition 13. Let $\mathcal{I} \subset 2^{\mathbb{N}}$ be a proper ideal in $\mathbb{N}$ and $(X, \rho)$ be a metric space. The sequence $x=\left(x_{n}\right)$ in $X$ is called to be $\mathcal{I}$-convergence to $\xi \in X$ if for each $\varepsilon>0$ the set $A(\varepsilon)=\left\{n \in \mathbb{N}: \rho\left(x_{n}, \xi\right) \geq \varepsilon\right\} \in \mathcal{I}$.

Definition 14. A sequence $x=\left\{x_{n}\right\}_{n \in \mathbb{N}}$ in $X$ is called to be $\mathcal{I}^{*}$-convergent to $\xi \in X$ iff there exists a set

$$
K \in \mathcal{F}(\mathcal{I}), K=\left\{k_{1}<k_{2}<\ldots<k_{p}<\ldots\right\} \subset \mathbb{N}
$$

such that $\lim _{p \rightarrow \infty} \rho\left(x_{k_{p}}, \xi\right)=0$.

Definition 15. ( [27]) Let $(X, \rho)$ be a linear metric space. If for every $\varepsilon>0$ there exists $N=N(\bar{\varepsilon})$ such that $A(\varepsilon)=\left\{n \in \mathbb{N}: \rho\left(x_{n}, x_{N}\right) \geq \varepsilon\right\} \in \mathcal{I}$, a sequence $x=\left(x_{n}\right)$ in $X$ is called an $\mathcal{I}$-Cauchy sequence in $X$

Definition 16. ( [27) Let $(X, \rho)$ be a linear metric space. If there exists a set $K=\left\{k_{1}<k_{2}<\ldots<k_{p}<\ldots\right\} \subset \mathbb{N}, K \in \mathcal{F}(\mathcal{I})$ such that $\lim _{p, r \rightarrow \infty} \rho\left(x_{k_{p}}, x_{k_{r}}\right)=0, a$ sequence $x=\left(x_{n}\right)$ in $X$ is called to be $\mathcal{I}^{*}$-Cauchy sequence .

In 2008, the term of ideal convergence for triple sequences used first time by Şahiner and Tripathy 31 in 2008.

Definition 17. A real triple sequence $\left(x_{n k l}\right)$ is called to be $\mathcal{I}$-convergent to $\ell$ if for every $\varepsilon>0$,

$$
\left\{(n, k, l) \in \mathbb{N}^{3}:\left|x_{n k l}-\ell\right| \geq \varepsilon\right\} \in \mathcal{I}_{3} .
$$

In this case, one writes $\mathcal{I}_{3}-\lim x_{n k l}=\ell$.

Throughout the paper we consider the ideals of $2^{\mathbb{N}}$ by $\mathcal{I}$; the ideals of $2^{\mathbb{N}^{2}}$ by $\mathcal{I}_{2}$ and the ideals of $2^{\mathbb{N}^{3}}$ by $\mathcal{I}_{3}$. 


\section{2. $\mathcal{I}_{3}$-CONVERGENCE IN FMS}

The terms of ideal convergence of triple sequences with a FMS were presented in this section.

Definition 18. Let $\mathcal{I}_{3}$ be a nontrivial ideal of $\mathbb{N}^{3}$ and $(X, \mathcal{M}, *)$ be a FMS. A triple sequence $x=\left\{x_{j k l}\right\}$ of elements of $X$ is said to be $\mathcal{I}_{3}$-convergent to $\ell \in X$ if, for each $r \in(0,1)$ and each $t>0$,

$$
\left\{(j, k, l) \in \mathbb{N}^{3}: \mathcal{M}\left(x_{j k l}, \ell, t\right)>1-r\right\} \in \mathcal{F}\left(\mathcal{I}_{3}\right) .
$$

In this stution we prefer to write as $\mathcal{I}_{3}^{\mathcal{M}}-\lim x=\ell$.

Theorem 1. Let $(X, \mathcal{M}, *)$ be a FMS. Then, for each $r \in(0,1)$ and each $t>0$, the following expression were equivalent:

(i) $\left\{(j, k, l) \in \mathbb{N}^{3}: \mathcal{M}\left(x_{j k l}, \ell, t\right)>1-r\right\} \in \mathcal{F}\left(\mathcal{I}_{3}\right)$.

(ii) $\left\{(j, k, l) \in \mathbb{N}^{3}: \mathcal{M}\left(x_{j k l}, \ell, t\right) \leq 1-r\right\} \in \mathcal{I}_{3}$.

Theorem 2. Let $x=\left\{x_{j k l}\right\}$ be a triple sequence in a FMS $(X, \mathcal{M}, *)$. When $x=\left\{x_{j k l}\right\}$ is $\mathcal{I}_{3}$-convergent to $\ell_{1}$ and $\ell_{2}, \ell_{1}=\ell_{2}$.

Proof. Assume that $\mathcal{I}_{3}^{\mathcal{M}}-\lim x=\ell_{1}$ and $\mathcal{I}_{3}^{\mathcal{M}}-\lim x=\ell_{2}$. Let $\ell_{1}$ and $\ell_{2}$ be two distinct points in $X$ and $t>0$. In that case $0<\mathcal{M}\left(\ell_{1}, \ell_{2}, t\right)<1$. Let $1-\varepsilon \in$ $\left(\mathcal{M}\left(\ell_{1}, \ell_{2}, t\right), 1\right)$. For each $1-s \in(1-\varepsilon, 1)$, there exists $1-s$ such that $(1-s) *$ $(1-s) \geq 1-\varepsilon$. Let

$$
K_{\ell_{1}}=\left\{y \in X: \mathcal{M}\left(\ell_{1}, y, \frac{t}{2}\right)>1-s\right\}
$$

and

$$
K_{\ell_{2}}=\left\{y \in X: \mathcal{M}\left(\ell_{2}, y, \frac{t}{2}\right)>1-s\right\} .
$$

We claim that $K_{\ell_{1}} \cap K_{\ell_{2}}=\emptyset$. Really, if there exists $z \in K_{\ell_{1}} \cap K_{\ell_{2}}$, then we get

$$
\begin{aligned}
\mathcal{M}\left(\ell_{1}, \ell_{2}, t\right) & \geq \mathcal{M}\left(\ell_{1}, z, \frac{t}{2}\right) * \mathcal{M}\left(z, \ell_{2}, \frac{t}{2}\right) \\
& \geq(1-s) *(1-s) \geq 1-\varepsilon \\
& >\mathcal{M}\left(\ell_{1}, \ell_{2}, t\right)
\end{aligned}
$$

which is a contradiction. Since

$$
\left\{y \in X: \mathcal{M}\left(\ell_{2}, y, \frac{t}{2}\right)>1-s\right\} \subset\left\{x \in X: \mathcal{M}\left(x, \ell_{1}, \frac{t}{2}\right) \leq 1-s\right\}
$$

it follows that

$$
\begin{aligned}
& \left\{(j, k, l) \in \mathbb{N}^{3}: \mathcal{M}\left(x_{j k l}, \ell_{2}, \frac{t}{2}\right)>1-s\right\} \\
& \subseteq\left\{(j, k, l) \in \mathbb{N}^{3}: \mathcal{M}\left(x_{j k l}, \ell_{1}, \frac{t}{2}\right) \leq 1-s\right\} .
\end{aligned}
$$


By (1), we get

$$
\begin{aligned}
& \left\{(j, k, l) \in \mathbb{N}^{3}: \mathcal{M}\left(x_{j k l}, \ell_{2}, \frac{t}{2}\right)>1-s\right\} \in \mathcal{F}\left(\mathcal{I}_{3}\right) \\
& \subseteq\left\{(j, k, l) \in \mathbb{N}^{3}: \mathcal{M}\left(x_{j k l}, \ell_{1}, \frac{t}{2}\right) \leq 1-s\right\} \in \mathcal{I}_{3} .
\end{aligned}
$$

which is a contradiction. Therefore, we conclude that $\mathcal{I}_{3}^{\mathcal{M}}$-lim must be unique. So the desired result has been obtained.

Theorem 3. Let $(X, \mathcal{M}, *)$ be a FMS and $\mathcal{I}_{3}$ be an admissible ideal. When triple sequence $x=\left\{x_{j k l}\right\}$ in $X$ is convergent to $\ell, x=\left\{x_{j k l}\right\}$ ideal converges to $\ell$.

Proof. Let $\lim \left\{x_{j k l}\right\}=\ell$. Let $r \in(0,1)$ and $t>0$. Then there exists a positive integer $n_{0}$ such that

$$
\mathcal{M}\left(x_{j k l}, \ell, t\right)>1-r
$$

for all $j>n_{0}, k>n_{0}, l>n_{0}$. Since

$$
\begin{aligned}
K_{\mathcal{M}} & =\left\{(j, k, l) \in \mathbb{N}^{3}: \mathcal{M}\left(x_{j k l}, \ell, \varepsilon\right) \leq 1-r\right\} \\
& \subseteq \mathbb{N}^{3}-\left\{\left(j_{n_{0}+1}, k_{n_{0}+1}, l_{n_{0}+1}\right),\left(j_{n_{0}+2}, k_{n_{0}+2}, l_{n_{0}+2}\right), \ldots\right\}
\end{aligned}
$$

and the ideal $\mathcal{I}_{3}$ is admissible, this implies that $K_{\mathcal{M}} \in \mathcal{I}_{3}$. Therefore

$$
\left\{(j, k, l) \in \mathbb{N}^{3}: \mathcal{M}\left(x_{j k l}, \ell, \varepsilon\right)>1-r\right\} \in \mathcal{F}\left(\mathcal{I}_{3}\right),
$$

that is $\mathcal{I}_{3}$ - $\lim x=\ell$. We complete the proof.

We gave the term of $\mathcal{I}_{3}^{*}$-convergence of triple sequences with a FMS.

Definition 19. Let $(X, \mathcal{M}, *)$ be a FMS. We say that a triple sequence $x=$ $\left\{x_{j k l}\right\}$ in $X$ is said to be $\mathcal{I}_{3}^{*}$-convergence to $\ell \in X$ if there exists a subset $K=$ $\left\{\left(j_{m}, k_{m}, l_{m}\right): j_{1}<j_{2}<\ldots ; k_{1}<k_{2}<\ldots ; l_{1}<l_{2}<\ldots\right\}$ of $\mathbb{N}^{3}$ such that $K \in \mathcal{F}\left(\mathcal{I}_{3}\right)$ (i.e. $\left.\mathbb{N}^{3} \backslash K \in \mathcal{I}_{3}\right)$ and $\left\{x_{j_{m} k_{m} l_{m}}\right\}$ converges to $\ell$.

In this stution we prefer to write $\mathcal{I}_{3}^{*-\mathcal{M}} \lim x=\ell$.

Theorem 4. Let $(X, \mathcal{M}, *)$ be a FMS and $\mathcal{I}_{3}$ be an admissible ideal. If $\mathcal{I}_{3}^{*-\mathcal{M}} \lim x=$ $\ell$, then $\mathcal{I}_{3}^{\mathcal{M}} \lim x=\ell$.

Proof. Let $x=\left\{x_{j k l}\right\}$ be an $\mathcal{I}_{3}^{*}$-convergence to $\ell \in X$. Then by definition,

$$
K=\left\{\left(j_{m}, k_{m}, l_{m}\right): j_{1}<j_{2}<\ldots ; k_{1}<k_{2}<\ldots ; l_{1}<l_{2}<\ldots\right\}
$$

of $\mathbb{N}^{3}, K \in \mathcal{F}\left(\mathcal{I}_{3}\right)$ such that $\left\{x_{j_{m} k_{m} l_{m}}\right\}$ converges to $\ell$, so there exists $N \in \mathbb{N}$ such that all $r \in(0,1)$ and $t>0$,

$$
\mathcal{M}\left(x_{j_{m} k_{m} l_{m}}, \ell, t\right)>1-r, \forall m>N
$$

Since $\mathcal{I}_{3}$ is an admissible and

$$
\left\{\left(j_{m}, k_{m}, l_{m}\right) \in K: \mathcal{M}\left(x_{j_{m} k_{m} l_{m}}, \ell, t\right) \leq 1-r\right\}
$$


is contained in $\left\{j_{1}<j_{2}<\ldots<j_{N-1} ; k_{1}<k_{2}<\ldots<k_{N-1} ; l_{1}<l_{2}<\ldots<l_{N-1}\right\}$, we get

$$
\left\{\left(j_{m}, k_{m}, l_{m}\right) \in K: \mathcal{M}\left(x_{j_{m} k_{m} l_{m}}, \ell, t\right) \leq 1-r\right\} \in \mathcal{I}_{3} .
$$

In this case, when we let $H=\mathbb{N}^{3} \backslash K$ it is obvious that $H \in \mathcal{I}_{3}$ and

$$
\begin{aligned}
\{(j, k, l) & \left.\in \mathbb{N}^{3}: \mathcal{M}\left(x_{j k l}, \ell, t\right) \leq 1-r\right\} \subset H \cup \\
& \left\{j_{1}<j_{2}<\ldots<j_{N-1} ; k_{1}<k_{2}<\ldots<k_{N-1} ; l_{1}<l_{2}<\ldots<l_{N-1}\right\} .
\end{aligned}
$$

Hence

$$
\left\{j_{1}<j_{2}<\ldots<j_{N-1} ; k_{1}<k_{2}<\ldots<k_{N-1} ; l_{1}<l_{2}<\ldots<l_{N-1}\right\} \in \mathcal{I}_{3} .
$$

This means that

$$
\left\{(j, k, l) \in \mathbb{N}^{3}: \mathcal{M}\left(x_{j k l}, \ell, t\right)>1-r\right\} \in \mathcal{F}\left(\mathcal{I}_{3}\right),
$$

so, $\left\{x_{j k l}\right\}$ is $\mathcal{I}_{3}$-convergent to $\ell$. Hence the proof is complete.

In the example given below, the inverse of Theorem 4 is generally not provided.

Example 1. Let $a * b=a b$ and for all $a, b \in[0,1]$. If for every $x, y \in \mathbb{R}$ and $t>0$

$$
\mathcal{M}(x, y, t)=\frac{t}{t+|x-y|},
$$

then $(\mathbb{R}, \mathcal{M}, *)$ is a FMS with the usual metric |.|.

Let $\mathbb{N}^{3}=\cup_{i, j, l} \Delta_{i j l}$ be a decomposition of $\mathbb{N}^{3}$ such that, for any $(m, n, o) \in$ $\mathbb{N}^{3}$, each $\Delta_{i j l}$ contains infinitely many $(i, j, l)$ 's where $i \geq m, j \geq n, l \geq o$ and $\Delta_{i j l} \cap \Delta_{m n o}=\emptyset$ for $(i, j, l) \neq(m, n, o)$. Now we define a sequence $x_{m n o}=\frac{1}{i j l}$ if $(m, n, o) \in \Delta_{i j l}$. It is immediate to see that $\left\{x_{m n o}\right\}$ is not $\mathcal{I}_{3}^{*}$-convergence to 0 , but $\left\{x_{j k l}\right\}$ is $\mathcal{I}_{3}$-convergence to 0 .

The following definition was needed to prove that an $\mathcal{I}_{3}$-convergence come across with an $\mathcal{I}_{3}^{*}$-convergence for admissible ideals with property (AP3).

Definition 20. An admissible ideal $\mathcal{I}_{3} \subset 2^{\mathbb{N}^{3}}$ is said to satisfy the condition (AP3) if for every sequence $\left(A_{j}\right)_{j \in \mathbb{N}}$ of pairwise disjoint sets from $\mathcal{I}_{3}$ there are sets $B_{j} \subset \mathbb{N}$, $j \in \mathbb{N}$, such that the symmetric difference $A_{j} \Delta B_{j}$ is a finite set for every $j \in \mathbb{N}$ and $\cup_{j \in \mathbb{N}} B_{j} \in \mathcal{I}_{3}$.

Theorem 5. Let $(X, \mathcal{M}, *)$ be a FMS and $\mathcal{I}_{3}$ satisfy the condition (AP3). Then $\mathcal{I}_{3}$-convergence and $\mathcal{I}_{3}^{*}$-convergence coincide.

Proof. Let $x=\left\{x_{j k l}\right\}$ be an $\mathcal{I}_{3}^{*}$-convergence. Then, by Theorem 4, this sequence is $\mathcal{I}_{3}$-convergence where $\mathcal{I}_{3}$ need not have the (AP3) condition. Then, it is sufficient to prove that $x=\left(x_{j k l}\right)$ in $X$ is a $\mathcal{I}_{3}^{*}$-convergence to $\ell \in X$ under assumption that $\left(x_{j k l}\right)$ is an $\mathcal{I}_{3}$-convergence to $\ell \in X$. Hence by definition, for all $r \in(0,1)$ and $t>0$,

$$
\left\{(j, k, l) \in \mathbb{N}^{3}: \mathcal{M}\left(x_{j k l}, \ell, t\right)>1-r\right\} \in \mathcal{F}\left(\mathcal{I}_{3}\right) .
$$


Let

$$
K_{s}=\left\{(j, k, l) \in \mathbb{N}^{3}: 1-\frac{1}{s+1}>\mathcal{M}\left(x_{j k l}, \ell, t\right)>1-\frac{1}{s}\right\} .
$$

Then, for $t>0$ and each $s=1,2, \ldots$, we have that $\left\{K_{1}, K_{2}, \ldots\right\}$ is countable and $K_{s} \in \mathcal{I}_{3}$, and $K_{i} \cap K_{j}=\emptyset$ for $i \neq j$. By the property (AP3), there is countable class of sets $\left\{B_{1}, B_{2}, \ldots\right\} \in \mathcal{I}_{3}$ such that $K_{i} \Delta B_{i}$ is a finite set for every $i \in \mathbb{N}$ and $B=\cup_{i \in \mathbb{N}} B_{i} \in \mathcal{I}_{3}$. From the definition of the associate filter $\mathcal{F}\left(\mathcal{I}_{3}\right)$ there is a set $A \in \mathcal{F}\left(\mathcal{I}_{3}\right)$ such that $A=\mathbb{N}^{3} \backslash B$. To prove the theorem we should aim that the subsequence $\left\{x_{j k l}\right\}_{(j, k, l) \in A}$ converges to $\ell$. Let $\mu \in(0,1)$ and each $t>0$. Put $q=1,2, \ldots$ such that $\frac{1}{q}<\mu$. So

$$
\begin{aligned}
& \left\{(j, k, l) \in \mathbb{N}^{3}: \mathcal{M}\left(x_{j k l}, \ell, t\right) \leq 1-\mu\right\} \\
& \subset\left\{(j, k, l) \in \mathbb{N}^{3}: \mathcal{M}\left(x_{j k l}, \ell, t\right) \leq 1-\frac{1}{q}\right\} \\
& \subset \cup_{i=1}^{q+1} K_{i} .
\end{aligned}
$$

Since $K_{i} \Delta B_{i}, i=1,2, \ldots, q+1$ are finite, there exists $\left(j_{0}, k_{0}, l_{0}\right) \in \mathbb{N}^{3}$ such that

$$
\begin{aligned}
& \cup_{i=1}^{q+1} B_{i} \cap\left\{\left(j_{0}, k_{0}, l_{0}\right): j \geq j_{0}, k \geq k_{0} \text { and } l \geq l_{0}\right\} \\
& =\cup_{i=1}^{q+1} K_{i} \cap\left\{\left(j_{0}, k_{0}, l_{0}\right): j \geq j_{0}, k \geq k_{0} \text { and } l \geq l_{0}\right\} .
\end{aligned}
$$

If $j \geq j_{0}, k \geq k_{0}, l \geq l_{0}$ and $(j, k, l) \in A$ then $(j, k, l) \notin \cup_{i=1}^{q+1} B_{i}$. Therefore, by (3), we have $(j, k, l) \notin \cup_{i=1}^{q+1} K_{i}$. Thus, $j \geq j_{0}, k \geq k_{0}, l \geq l_{0}$ and $(j, k, l) \in A$, we have

$$
\mathcal{M}\left(x_{j k l}, \ell, t\right)>1-\mu .
$$

Since $\mu \in(0,1)$ is arbitrary, this shows that $\mathcal{I}_{3}^{*}-\lim x_{j k l}=\ell$.

\section{3. $\mathcal{I}_{3}$ - AND $\mathcal{I}_{3}^{*}$-CAUChy SEQUences on FMS}

Now, the terms of $\mathcal{I}_{3}$-Cauchy sequence and $\mathcal{I}_{3}^{*}$-Cauchy sequence was presented in FMS.

Definition 21. Let $(X, \mathcal{M}, *)$ be a FMS. A triple sequence $\left\{x_{j k l}\right\}$ in $X$ is called $\mathcal{I}_{3}$-Cauchy sequence if for every $\alpha \in(0,1)$ and $t>0$, there exists $N_{1}, N_{2}$ and $N_{3}$ such that for all $j, p \geq N_{1}, k, q \geq N_{2}, l, r \geq N_{3}$,

$$
\left\{(j, k, l) \in \mathbb{N}^{3}: \mathcal{M}\left(x_{j k l}, x_{p q r}, t\right)>1-\alpha\right\} \in \mathcal{F}\left(\mathcal{I}_{3}\right) .
$$

In this case, it is stated that $\left\{x_{j k l}\right\}$ is $\in \mathcal{I}_{3}^{\mathcal{M}}$-Cauchy.

Proceeding similarly, we get the following consequence.

Corollary 1. When a triple sequence in a FMS is Cauchy, it is $\mathcal{I}_{3}^{\mathcal{M}}$-Cauchy.

Definition 22. Let $(X, \mathcal{M}, *)$ be a FMS. A triple sequence $x=\left\{x_{j k l}\right\}$ in $X$ is called to be $\mathcal{I}_{3}^{*}$-Cauchy sequence in $X$ if there exists a subset $K=\left\{\left(j_{m}, k_{m}, l_{m}\right)\right.$ : 
$\left.j_{1}<j_{2}<\ldots ; k_{1}<k_{2}<\ldots ; l_{1}<l_{2}<\ldots\right\}$ of $\mathbb{N}^{3}$ such that $K \in \mathcal{F}\left(\mathcal{I}_{3}\right)$ and $\left\{x_{j_{m} k_{m} l_{m}}\right\}$ is a Cauchy sequence in $X$, i.e., there exists $N \in \mathbb{N}$ such that

$$
\mathcal{M}\left(x_{j k l}, x_{p q r}, t\right)>1-\alpha
$$

whenever $j \geq p \geq N, k \geq q \geq N, l \geq r \geq N$.

Here we can say that $\left\{x_{j k l}\right\}$ is $\in \mathcal{I}_{3}^{*-\mathcal{M}}$-Cauchy.

Since the next theorems are respectively analogues to Theorems 4 and 5 , it can be proved on same methods.

Theorem 6. Let $(X, \mathcal{M}, *)$ be a FMS and $\mathcal{I}_{3}$ be an admissible ideal. When a triple sequence $\left\{x_{j k l}\right\}$ is $\mathcal{I}_{3}^{*-\mathcal{M}}$-Cauchy, it is $\mathcal{I}_{3}^{\mathcal{M}}$-Cauchy.

Theorem 7. Let $(X, \mathcal{M}, *)$ be a FMS and $\mathcal{I}_{3}$ satisfy the condition (AP3). When a triple sequence $\left\{x_{j k l}\right\}$ is $\mathcal{I}_{3}^{\mathcal{M}}$-Cauchy, it is also $\mathcal{I}_{3}^{*-\mathcal{M}}$-Cauchy.

Therefore, we now present the following theorem.

Theorem 8. Let $\left\{x_{j k l}\right\}$ be a triple sequence in a FMS $(X, \mathcal{M}, *)$ and $\mathcal{I}_{3}$ be an arbitrary admissible ideal with property (AP3). Then $\mathcal{I}_{3}^{\mathcal{M}}-\lim x=\ell$ implies that $\left\{x_{j k l}\right\}$ is an $\mathcal{I}_{3}^{\mathcal{M}}$-Cauchy sequence.

Proof. Let $\mathcal{I}_{3}^{\mathcal{M}}-\lim x=\ell$. Then for every $r \in(0,1)$ and $t>0$,

$$
\left\{(j, k, l) \in \mathbb{N}^{3}: \mathcal{M}\left(x_{j k l}, \ell, t\right)>1-r\right\} \in \mathcal{F}\left(\mathcal{I}_{3}\right) .
$$

Let $\alpha \in(0,1)$ and $t>0$. Then there exists $\alpha_{1} \in(0, \alpha)$ such that $\left(1-\alpha_{1}\right) *(1-$ $\left.\alpha_{1}\right)>1-\alpha$. According to Theorem 5 and Definition 20, there exists a subset $A=\left\{\left(j_{m}, k_{m}, l_{m}\right): j_{1}<j_{2}<\ldots ; k_{1}<k_{2}<\ldots ; l_{1}<l_{2}<\ldots\right\}$ of $\mathbb{N}^{3}$ such that $A \in \mathcal{F}\left(\mathcal{I}_{3}\right)$ and $\left\{x_{j_{m} k_{m} l_{m}}\right\}$ converges to $\ell$. Thus there exists $N \in \mathbb{N}$ such that

$$
\mathcal{M}\left(x_{j_{m} k_{m} l_{m}}, \ell, \frac{t}{2}\right)>1-\alpha_{1} \text { for every } m>N \text {. }
$$

Let $(p, q, r) \in\left\{(j, k, l) \in \mathbb{N}^{3}: \mathcal{M}\left(x_{j k l}, \ell, \frac{t}{2}\right)>1-\alpha_{1}\right\}$. Then

$$
\begin{aligned}
\mathcal{M}\left(x_{p q r}, x_{j_{m} k_{m} l_{m}}, t\right) & \geq \mathcal{M}\left(x_{p q r}, \ell, t / 2\right) * \mathcal{M}\left(x_{j_{m} k_{m} l_{m}}, \ell, t / 2\right) \\
& \geq\left(1-\alpha_{1}\right) *\left(1-\alpha_{1}\right)>1-\alpha .
\end{aligned}
$$

Hence $(p, q, r) \in\left\{(j, k, l) \in \mathbb{N}^{3}: \mathcal{M}\left(x_{j k l}, x_{j_{m} k_{m} l_{m}}, \frac{t}{2}\right)>1-\alpha_{1}\right\}$. It follows that

$$
\begin{aligned}
& \left\{(j, k, l) \in \mathbb{N}^{3}: \mathcal{M}\left(x_{j k l}, \ell, \frac{t}{2}\right)>1-\alpha_{1}\right\} \\
& \subseteq\left\{(j, k, l) \in \mathbb{N}^{3}: \mathcal{M}\left(x_{j k l}, x_{j_{m} k_{m} l_{m}}, t\right)>1-\alpha_{1}\right\} .
\end{aligned}
$$

Since $\left\{(j, k, l) \in \mathbb{N}^{3}: \mathcal{M}\left(x_{j k l}, \ell, \frac{t}{2}\right)>1-\alpha_{1}\right\} \in \mathcal{F}\left(\mathcal{I}_{3}\right)$ and (4), we get that

$$
\left\{(j, k, l) \in \mathbb{N}^{3}: \mathcal{M}\left(x_{j k l}, x_{j_{m} k_{m} l_{m}}, t\right)>1-\alpha_{1}\right\} \in \mathcal{F}\left(\mathcal{I}_{3}\right) .
$$

This indicate that the triple sequence $\left\{x_{j k l}\right\}$ in $X$ is an $\mathcal{I}_{3}^{\mathcal{M}}$-Cauchy sequence. 
Remark 1. But the converse of the above theorem is not necessarily true, i.e. $\mathcal{I}_{3}^{\mathcal{M}}$-Cauchy sequence does not imply $\mathcal{I}_{3}^{\mathcal{M}}$-lim $x=\ell$. This can be illustrated by the example given below.

Example 2. Let $\mathcal{I}_{3}^{\mathcal{M}}(\delta)=\left\{A \subset \mathbb{N}^{3}: \delta_{3}(A)=0\right\}$ and $X=\left\{x_{j k l}:(j, k, l) \in \mathbb{N}^{3}\right\}$, where $x_{j k l}=1-\frac{1}{(j+1)(k+1)(l+1)}(j, k, l \in \mathbb{N})$ and $a * b=\min \{a, b\}$ for all $a, b \in[0,1]$, and let $\mathcal{M}$ be a fuzzy set on $X^{2} \times(0, \infty)$ define as follows $\mathcal{M}(x, y, t)$ to be 1 for $x=y$ and $\min \{x, y\}$ otherwise, for all $x, y \in X$ and $t>0$. Hence $(X, \mathcal{M}, *)$ is a FMS and triple sequence $\left\{x_{j k l}\right\}$ in $(X, \mathcal{M}, *)$ is $\mathcal{I}_{3}^{\mathcal{M}}$-Cauchy, but it is not $\mathcal{I}_{3}^{\mathcal{M}}$-convergent.

Let $\alpha \in(0,1)$ and $t>0$. Therefore there exists $p, q, r \in \mathbb{N}$ such that $\frac{1}{(p+1)(q+1)(r+1)}<\alpha$. Hence

$$
\mathcal{M}\left(x_{j k l}, x_{p q r}, t\right)=x_{p q r}=1-\frac{1}{(p+1)(q+1)(r+1)}>1-\alpha
$$

for all $j>p, k>q, l>r$. Thus

$$
\left\{(j, k, l) \in \mathbb{N}^{3}: \mathcal{M}\left(x_{j k l}, x_{p q r}, t\right)>1-\alpha\right\} \in \mathcal{F}\left(\mathcal{I}_{3}(\delta)\right) .
$$

which means that $\left\{x_{j k l}\right\}$ is $\mathcal{I}_{3}^{\mathcal{M}}$-Cauchy sequence. Let $\ell \in X$. Then there exists $p, q, r \in \mathbb{N}$ such that $\ell=x_{p q r}=1-\frac{1}{(p+1)(q+1)(r+1)}$. Now, fix $t_{0}=\alpha_{0}=$ $\frac{1}{3(p+1)(q+1)(r+1)}$. Then

$$
\mathcal{M}\left(x_{j k l}, \ell, t_{0}\right)=\mathcal{M}\left(x_{j k l}, x_{p q r}, t_{0}\right)=x_{p q r}=1-\frac{1}{(p+1)(q+1)(r+1)} \leq 1-\alpha_{0}
$$

for all $j>p, k>q, l>r$. Hence

$$
\left\{(j, k, l) \in \mathbb{N}^{3}: \mathcal{M}\left(x_{j k l}, \ell, t_{0}\right) \leq 1-\alpha_{0}\right\} \in \mathcal{F}\left(\mathcal{I}_{3}(\delta)\right)
$$

which implies that

$$
\left\{(j, k, l) \in \mathbb{N}^{3}: \mathcal{M}\left(x_{j k l}, \ell, t_{0}\right)>1-\alpha_{0}\right\} \in \mathcal{I}_{3}(\delta) .
$$

So $\left\{x_{j k l}\right\}$ is not $\mathcal{I}_{3}^{\mathcal{M}}$-convergent.

As a note, all these findings imply the similar theorems for ideal and statistically convergence and Cauchy sequences which are investigated in 19] and 26.

Author Contribution Statements The authors contributed equally to this work. All authors read and approved the final copy of this paper.

Declaration of Competing Interests The authors declare that they have no known competing financial interest or personal relationships that could have appeared to influence the work reported in this paper. 


\section{REFERENCES}

[1] Das, P., Kostyrko, P., Wilczyski, W., Malik, P., $\mathcal{I}$ and $\mathcal{I}^{*}$-convergence of double sequences, Math. Slovaca, 58(5) (2008), 605-620. https://doi.org/10.2478/s12175-008-0096-x

[2] Engelking, R., General Topology, PWN-Polish Science Publishers, Warsaw, 1977.

[3] Erceg, M. A., Metric spaces in fuzzy set theory, J. Math. Anal. Appl., 69(1) (1979), 205-230. https://doi.org/10.1016/0022-247X(79)90189-6

[4] Fast, H., Sur la convergence statistique, Colloq. Math., 2(3-4) (1951), 241-244.

[5] George, A., Veeramani, P., On some results in fuzzy metric spaces, Fuzzy Sets and Systems, 64(3) (1994), 395-399. https://doi.org/10.1016/0165-0114(94)90162-7

[6] George, A., Veeramani, P., On some results of analysis for fuzzy metric spaces, Fuzzy Sets and Systems, 90(3) (1997), 365-368. https://doi.org/10.1016/S0165-0114(96)00207-2

[7] Gregori, V., López-Crevillén, A., Morillas, S., Sapena, A., On convergence in fuzzy metric spaces, Topology Appl., 156(18) (2009), 3002-3006. https://doi.org/10.1016/j.topol.2008.12.043

[8] Gregori, V., Miñana, J. J., Std-convergence in fuzzy metric spaces, Fuzzy Sets and Systems, 267 (2015), 140-143. https://doi.org/10.1016/j.fss.2014.05.007

[9] Gregori, V., Miñana, J. J., Morillas, S., A note on convergence in fuzzy metric spaces, Iran J. Fuzzy Syst., 11(4) (2014), 75-85. https://doi.org/10.22111/IJFS.2014.1625

[10] Gregori, V., Miñana, J. J., Morillas, S., Sapena, A., Cauchyness and convergence in fuzzy metric spaces, Rev. R. Acad. Cienc. Exactas Fís. Nat. Ser. A Mat. RACSAM, 111(1) (2017), 25-37. https://doi.org/10.1007/s13398-015-0272-0

[11] Gürdal, M., Some types of convergence, Doctoral Dissertation, Süleyman Demirel University, Isparta, 2004.

[12] Gürdal, M., Açık, I., On I-Cauchy sequences in 2-normed spaces, Math. Inequal. Appl., 11(2) (2008), 349-354. https://doi.org/10.7153/mia-11-26

[13] Gürdal, M., Huban, M. B., On $\mathcal{I}$-convergence of double sequences in the topology induced by random 2-norms, Mat. Vesnik, 66(1) (2014), 73-83.

[14] Gürdal, M., Sarı, N., Savaş, E., $A$-statistically localized sequences in $n$-normed spaces, Commun. Fac. Sci. Univ. Ank. Ser. A1 Math. Stat., 69(2) (2020), 1484-1497. https://doi.org/10.31801/cfsuasmas.704446

[15] Gürdal, M., Şahiner, A., Extremal I-limit points of double sequences, Appl. Math. E-Notes, 8 (2008), 131-137.

[16] Hazarika, B., Alotaibi, A., Mohiuddine, S. A., Statistical convergence inmeasure for double sequences of fuzzy-valued functions, Soft Comput., 24 (2020), 6613-6622. https://doi.org/10.1007/s00500-020-04805-y

[17] Kaleva, O., Seikkala, S., On fuzzy metric spaces, Fuzzy Sets and Systems, 12(3) (1984), 215-229. https://doi.org/10.1016/0165-0114(84)90069-1

[18] Kramosil, I., Michalek, J., Fuzzy metric and statistical metric spaces, Kybernetika, 11(5) (1975), 336-344.

[19] Li, C., Zhang, Y., Zhang, J., On statistical convergence in fuzzy metric spaces, J. Intell. Fuzzy Systems, 39(3) (2020), 3987-3993. https://doi.org/10.3233/JIFS-200148

[20] Kostyrko, S., Ŝalát, T., Wilczyňski, W., I-convergence, Real Anal. Exchange, 26(2) (2000), 669-686.

[21] Mohiuddine, S. A., Asiri, A., Hazarika, B., Weighted statistical convergence through difference operator of sequences of fuzzy numbers with application to fuzzy approximation theorems, Int. J. Gen. Syst., 48(5) (2019), 492-506. https://doi.org/10.1080/03081079.2019.1608985

[22] Mohiuddine, S. A., Hazarika, B., Some classes of ideal convergent sequences and generalized difference matrix operator, Filomat, 31(6) (2017), 1827-1834. https://doi.org/10.2298/FIL1706827M 
[23] Mohiuddine, S. A., Hazarika, B., Alotaibi, A., On statistical convergence of double sequences of fuzzy valued functions, J. Intell. Fuzzy Systems, 32 (2017), 4331-4342. https://doi.org/10.3233/JIFS-16974

[24] Mohiuddine, S. A., Hazarika, B., Alghamdi, M. A., Ideal relatively uniform convergence with Korovkin and Voronovskaya types approximation theorems, Filomat, 33(14) (2019), 45494560. https://doi.org/10.2298/FIL1914549M

[25] Mursaleen, M., Mohiuddine, S. A., On ideal convergence in probabilistic normed spaces, Math. Slovaca, 62(1) (2012), 49-62. https://doi.org/10.2478/s12175-011-0071-9

[26] Mursaleen, M., Mohiuddine, S. A., Osama Edely, H. H., On the ideal convergence of double sequences in intuitionistic fuzzy normed spaces, Comput. Math. Appl., 59(2) (2010), 603-611. https://doi.org/10.1016/j.camwa.2009.11.002

[27] Nabiev, A., Pehlivan, S., Gürdal, M., On I-Cauchy sequence, Taiwanese J. Math., 11(2) (2007), 569-576.

[28] Nabiev, A. A., Savaş, E., Gürdal, M., I-localized sequences in metric spaces, Facta Univ. Ser. Math. Inform., 35(2) (2020), 459-469. https://doi.org/10.22190/FUMI2002459N

[29] Rath, D., Tripathy, B. C., On statistically convergence and statistically Cauchy sequences, Indian J. Pure Appl. Math., 25(4) (1994), 381-386.

[30] Şahiner, A. Gürdal, M., Düden, F. K., Triple sequences and their statistical convergence, Selçuk J. Appl. Math., 8(2) (2007), 49-55.

[31] Şahiner, A., Tripathy, B. C., Some $\mathcal{I}$-related properties of triple sequences, Selçuk J. Appl. Math., 9(2) (2008), 9-18.

[32] Savaş, E., Gürdal, M., Certain summability methods in intuitionistic fuzzy normed spaces, J. Intell. Fuzzy Systems, 27(4) (2014), 1621-1629. https://doi.org/10.3233/IFS-141128

[33] Savaş, E., Gürdal, M., Generalized statistically convergent sequences of functions in fuzzy 2normed spaces, J. Intell. Fuzzy Systems, 27 (2014), 2067-2075. https://doi.org/10.3233/IFS141172

[34] Savaş, E., Gürdal, M., I-statistical convergence in probabilistic normed spaces, Politehn. Univ. Bucharest Sci. Bull. Ser. A Appl. Math. Phys., 77(4) (2015), 195-204.

[35] Savaş, E., Gürdal, M., Ideal convergent function sequences in random 2-normed spaces, Filomat, 30(3) (2016), 557-567. https://doi.org/10.2298/FIL1603557S

[36] Savaş, E., Mursaleen, M., On statistically convergent double sequences of fuzzy numbers, Inform. Sci., 162(3-4) (2004), 183-192. https://doi.org/10.1016/j.ins.2003.09.005

[37] Savaş, E., Yamancı, U., Gürdal, M., I-lacunary statistical convergence of weighted $g$ via modulus functions in 2-normed spaces, Commun. Fac. Sci. Univ. Ank. Ser. A1 Math. Stat., 68(2) (2019), 2324-2332. https://doi.org/10.31801/cfsuasmas.573396

[38] Schweizer, B., Sklar, A., Statistical metric spaces, Pacific J. Math., 10(1) (1960), 314-334.

[39] Yamancı, U., Gürdal, M., Std-statistical convergence in intuitionistic fuzzy normed spaces, Notes on Intuitionistic Fuzzy Sets, 22(2) (2016), 52-58.

[40] Yamancı, U., Savaş, E., Gürdal, M., I-localized sequence in two normed spaces, Malaysian J. Math. Sci., 14(3) (2020), 491-503.

[41] Zadeh, L.A., Fuzzy sets, Inform. Control, 8(3) (1965), 338-353. https://doi.org/10.1016/S0019-9958(65)90241-X 